\title{
Photoexposition discriminates Notch 1 expression in human cutaneous squamous cell carcinoma
}

\author{
John Panelos ${ }^{1}$, Francesca Tarantini ${ }^{2}$, Milena Paglierani ${ }^{3}$, Claudia Di Serio ${ }^{2}$, Vincenza Maio ${ }^{3}$, \\ Silvia Pellerito ${ }^{2}$, Nicola Pimpinelli ${ }^{4}$, Marco Santucci ${ }^{3}$ and Daniela Massi ${ }^{3}$ \\ ${ }^{1}$ Department of Pathology, University of Ioannina Medical School, Ioannina, Greece; ${ }^{2}$ Department of Critical \\ Care Medicine and Surgery, University of Florence, Florence, Italy; ${ }^{3}$ Department of Human Pathology \\ and Oncology, University of Florence, Florence, Italy and ${ }^{4}$ Department of Dermatological Sciences, \\ University of Florence, Florence, Italy
}

\begin{abstract}
The Notch signaling pathway may play opposing roles in cancer. It can be oncosuppressive or protumoral, depending on the cellular and tissue context. In skin cancer, Notch 1 expression is downregulated, thus supporting the hypothesis of an oncosuppressive role in cutaneous carcinomas. However, as members of the Notch family undergo downregulation upon exposure to UV irradiation, we wondered whether Notch 1 expression in skin carcinomas may be governed by additional factors, including UV exposure. We investigated the expression of Notch 1 and its ligands, Jagged 1, Jagged 2 and Delta-like 1, by immunohistochemistry in a series of premalignant and invasive cutaneous carcinomas, including 4 solar keratoses, 5 Bowen's disease, 5 squamous cell carcinomas on sun-exposed skin, 6 squamous cell carcinomas on sun-protected genital skin and 14 basal cell carcinomas of different histotypes (nodular, superficial type, sclerodermiform/infiltrating and baso-squamous). Expression of Notch 1 was decreased in solar keratoses and invasive squamous cell carcinomas localized on sun-exposed skin. In contrast, marked Notch 1 staining was observed in extragenital Bowen's disease as well as in genital (penile) human papilloma virus-related in situ and invasive squamous cell carcinomas. A diffuse Notch 1 staining was detected in nodular and superficial basal cell carcinomas while sclerodermiform/infiltrating and baso-squamous basal cell carcinomas showed a low to absent Notch 1 expression. Jagged 1, Jagged 2 and Delta-like 1 proteins were expressed in all tissues examined. Present findings show divergent expression of Notch 1 in skin cancer, depending on anatomical site and tumor histotype. Thus, whereas in UV-related squamous cell photocarcinogenesis Notch 1 downregulation could mirror a tumor suppressor function of the receptor, in sun-protected squamous cell carcinomas Notch 1 was upregulated. Furthermore, Notch 1 expression was minimal in basal cell carcinoma subtypes correlated with risk of recurrence (sclerodermiform/infiltrating and baso-squamous) in comparison with nodular and superficial types.
\end{abstract}

Modern Pathology (2008) 21, 316-325; doi:10.1038/modpathol.3801007; published online 11 January 2008

Keywords: Notch 1; squamous cell carcinoma; basal cell carcinoma; solar keratosis; solar UV; skin

Non-melanoma skin cancer, although associated to low metastatic potential and mortality rate, still represents a relevant public health problem because of its steadily increasing incidence. ${ }^{1,2}$ Non-melanoma skin cancer account for $>90 \%$ of all cutaneous malignant tumors, and approximately $70 \%$ of

Correspondence: Professor D Massi, MD, Dipartimento di Patologia Umana ed Oncologia, Università degli Studi di Firenze, Viale GB Morgagni 85, I-50134 Firenze, Italy.

E-mail: Daniela.massi@UNIFI.IT

Received 22 August 2007; revised 19 November 2007; accepted 26 November 2007; published online 11 January 2008 them are basal cell carcinomas. Basal cell carcinomas develop predominantly on sun-exposed skin, in individuals with fair complexion and prone to sunburn. Squamous cell carcinomas arise on sundamaged skin of elderly people. However, despite a major association with sun exposure, squamous cell carcinomas may also originate on sun-protected skin.

Increasing evidence indicates that Notch signaling has a major contribution in the regulation of cell-fate decisions, including self-renewal of adult stem cells and differentiation of precursors along a specific cell lineage. ${ }^{3}$ In mammals, the Notch system consists of 
four transmembrane receptors (Notch 1-4) and five transmembrane ligands (Jagged 1 and Jagged 2, Delta-like 1, -3 and -4). ${ }^{4,5}$ Notch signaling is initiated by receptor-ligand interaction between neighboring cells, resulting in proteolytic cleavage of the receptor by TACE (TNF- $\alpha$-converting enzyme) and subsequently by the $\gamma$-secretase/presenilin complex. This processing results in the release of the Notch intracellular domain (Notch IC) from the plasma membrane, which translocates to the nucleus, where often associates with the DNAbinding transcription factor, CLS (acronym for CBF1, LAG-1 and $S u(H)) .{ }^{6}$ The complex of Notch IC with the transcription factor CLS has been shown to transactivate the basic helix-loop-helix transcription factor Hes1, thus regulating downstream target genes. ${ }^{7}$

Owing to the complexity of the Notch signaling pathway, it is difficult to predict the net outcome of Notch activation in the setting of cancer. Substantial evidence suggests that Notch signaling can act as either a tumor promoter or a suppressor, depending on the cell type and tissue context, level of expression and potential cross talk with other signaling cascades. ${ }^{8}$ In cutaneous melanoma, Notch activation represents an early event in melanocytic tumor growth and upregulation of Notch signaling may sustain tumor progression. ${ }^{9}$ In contrast, in nonmelanoma skin cancer, namely keratinocytic tumors, the Notch pathway seems to behave differently. Notch 1 triggers differentiation and exerts growth-inhibitory functions in murine ${ }^{10}$ and human keratinocytes, in vitro. ${ }^{11}$ Genetic deletion of Notch signaling in primary human keratinocytes is sufficient, together with activated ras, to cause aggressive squamous cell carcinoma formation. ${ }^{12}$ Notch 1 tumor suppressor function has been attributed to increased p21WAF/Cip1 expression with subsequent downmodulation of specific Wnt family members. ${ }^{13}$ Furthermore, it has been recently shown that Notch 1 gene is a p53 target in human keratinocytes, with a tumor suppressor role through negative regulation of the Rho effectors ROCK $1 / 2$ and MRCK $\alpha$ kinases. ${ }^{12}$ Finally, additional evidence for an oncosuppressive role of Notch 1 derives from in vivo mouse studies showing that Notch 1 ablation results in the development of skin tumors and facilitation of chemical-induced skin carcinogenesis. ${ }^{14,15}$

There is evidence that Notch 4 is downregulated in cultured human keratinocytes upon UVB irradiation. ${ }^{16}$ Thus, the hypothesis can be advanced that, independently from tumor development, additional factors may contribute to modulation of Notch signaling in skin, including UV irradiation. For this purpose, we have compared the immunohistochemical expression of Notch 1, and three ligands, Jagged 1, Jagged 2 and Delta-like 1, in human tissue specimens from sun-exposed precancerous and cancerous skin lesions and sun-protected skin cancer.

\section{Materials and methods}

\section{Specimen Selection}

The study series included human tissue samples retrieved from the archive of the Department of Human Pathology and Oncology, University of Florence. The series was composed of solar/actinic keratoses $(n=4)$, Bowen's disease (in situ squamous cell carcinoma) $(n=5)$, cutaneous squamous cell carcinomas on sun-exposed skin $(n=5)$, cutaneous squamous cell carcinomas on sun-protected skin $(n=6)$ and cutaneous basal cell carcinomas $(n=14)$. Basal cell carcinomas were representative of different histotypes, including nodular $(n=3)$, superficial type $(n=4)$, sclerodermiform $(n=3)$ and basosquamous basal cell carcinomas $(n=4)$. All squamous cell carcinoma and most basal cell carcinoma tissue samples on sun-exposed skin included both tumor and adjacent photodamaged skin. All squamous cell carcinomas on sun-protected skin included both tumor and adjacent normal skin.

Patients' data, including age, sex and anatomic site of tumor, were retrospectively collected and are reported in Table 1.

\section{Immunohistochemistry}

Sections of $4 \mu \mathrm{m}$ thickness were cut from tissue blocks of formalin-fixed, paraffin-embedded samples. Slides were dewaxed in Bio-Clear (Bio-Optica, Milano, Italy) and hydrated with grade ethanol concentrations. Tissue sections were labeled with the following antibodies: goat anti-human Notch 1 (sc-6014), goat anti-human Jagged 1 (sc-6011), goat anti-human Jagged 2 (sc-8157) and rabbit antihuman Delta-like 1 (sc-9102) (Santa Cruz Biotechnology Inc.). Briefly, antigen retrieval was performed by microwave pretreatment (Microwave MicroMED $\mathrm{T} / \mathrm{T}$ Mega; Milestone, Bergamo, Italy) in $1 \mathrm{mM}$ EDTA, pH 8.0, for $30 \mathrm{~min}$; endogenous peroxidase activity was blocked by immersing slides in distilled water, containing $3.0 \%$ hydrogen peroxide, for 20 min. Nonspecific antigen sites were blocked with normal horse serum (UltraVision kit; LabVision, Fremont, CA, USA), then the sections were incubated with primary antibodies at the indicated dilutions. Staining was achieved using appropriate biotin-conjugated anti-goat secondary antibody (IgG; Biomedicals, Verona, Italy) and a biotinylated goat anti-rabbit (UltraVision kit), followed by streptavidin-peroxidase (UltraVision kit).

Selected specimens (two solar keratoses, two Bowen's disease, seven squamous cell carcinomas and four basal cell carcinomas) were immunohistochemically evaluated for p53 expression. Sections were treated with an antigen-retrieval procedure by heating in a microwave oven for $30 \mathrm{~min}$, in $10 \mathrm{mmol} / \mathrm{l}$ citrate buffer ( $\mathrm{pH}$ 6.0). They were incubated with the monoclonal antibody DO7 (1:40 dilution; Dako, Glostrup, Denmark) for $60 \mathrm{~min}$ at $20^{\circ} \mathrm{C}$, followed by 
Table 1 Distribution of Notch 1, Jagged 1, Jagged 2 and Delta-like 1 expression in solar keratoses, Bowen's disease, squamous cell carcinoma and basal cell carcinoma $(n=34)$

\begin{tabular}{|c|c|c|c|c|c|c|c|c|}
\hline No. & Diagnosis & Age (years) & Sex & Site & Notch 1 & Jag 1 & Jag 2 & $D l-1$ \\
\hline 1 & Solar keratosis & 61 & $\mathrm{~F}$ & Nose & 0 & $3+$ & $3+$ & $3+$ \\
\hline 2 & Solar keratosis & 79 & M & Forearm & $1+$ & $3+$ & $3+$ & $3+$ \\
\hline 3 & Solar keratosis & 75 & $\mathrm{M}$ & Cheek & $1+$ & $3+$ & $3+$ & $3+$ \\
\hline 4 & Solar keratosis & 60 & $\mathrm{M}$ & Scalp & 0 & $3+$ & $3+$ & $3+$ \\
\hline 5 & Bowen's disease & 85 & $\mathrm{M}$ & Leg & $3+$ & $3+$ & $3+$ & $3+$ \\
\hline 6 & Bowen's disease & 76 & $\mathrm{~F}$ & Face & $3+$ & $3+$ & $3+$ & $3+$ \\
\hline 7 & Bowen's disease & 53 & $\mathrm{M}$ & Leg & $3+$ & $3+$ & $3+$ & $3+$ \\
\hline 8 & Bowen's disease & 89 & $\mathrm{M}$ & Thigh & $3+$ & $3+$ & $3+$ & $3+$ \\
\hline 9 & Bowen's disease & 62 & $\mathrm{M}$ & Trunk & $3+$ & $3+$ & $3+$ & $3+$ \\
\hline 10 & SCC (sun-exposed site) & 92 & $\mathrm{M}$ & Cheek & 0 & $3+$ & $3+$ & $3+$ \\
\hline 11 & SCC (sun-exposed site) & 83 & $\mathrm{M}$ & Forehead & $2+$ & $3+$ & $3+$ & $3+$ \\
\hline 12 & SCC (sun-exposed site) & 91 & $\mathrm{M}$ & Scalp & 0 & $3+$ & $3+$ & $3+$ \\
\hline 13 & SCC (sun-exposed site) & 82 & $\mathrm{~F}$ & Nose & $2+$ & $3+$ & $3+$ & $3+$ \\
\hline 14 & SCC (sun-exposed site) & 80 & $\mathrm{M}$ & Face & $1+$ & $3+$ & $3+$ & $3+$ \\
\hline 15 & SCC (sun-protected site) & 57 & $\mathrm{M}$ & Glans & $3+$ & $3+$ & $3+$ & $3+$ \\
\hline 16 & SCC (sun-protected site) & 57 & $\mathrm{M}$ & Thigh & $3+$ & $3+$ & $3+$ & $3+$ \\
\hline 17 & SCC (sun-protected site) & 93 & $\mathrm{M}$ & Penis & $3+$ & $3+$ & $3+$ & $3+$ \\
\hline 18 & SCC (sun-protected site) & 72 & $\mathrm{M}$ & Penis & $3+$ & $3+$ & $3+$ & $3+$ \\
\hline 19 & SCC (sun-protected site) & 81 & $\mathrm{M}$ & Penis & $3+$ & $3+$ & $3+$ & $3+$ \\
\hline 20 & SCC (sun-protected site) & 43 & $\mathrm{M}$ & Penis & $3+$ & $3+$ & $3+$ & $3+$ \\
\hline 21 & BCC (nodular type) & 73 & $\mathrm{M}$ & Cheek & $3+$ & $3+$ & $3+$ & $3+$ \\
\hline 22 & BCC (nodular type) & 73 & $\mathrm{~F}$ & Face & $3+$ & $3+$ & $3+$ & $3+$ \\
\hline 23 & BCC (nodular type) & 35 & $\mathrm{~F}$ & Trunk & $3+$ & $3+$ & $3+$ & $3+$ \\
\hline 24 & BCC (superficial type) & 83 & $\mathrm{M}$ & Shoulder & $2+$ & $3+$ & $3+$ & $3+$ \\
\hline 25 & BCC (superficial type) & 67 & $\mathrm{M}$ & Back & $3+$ & $3+$ & $3+$ & $3+$ \\
\hline 26 & BCC (superficial type) & 54 & $\mathrm{M}$ & Face & $3+$ & $3+$ & $3+$ & $3+$ \\
\hline 27 & BCC (superficial type) & 66 & $\mathrm{M}$ & Back & $3+$ & $3+$ & $3+$ & $3+$ \\
\hline 28 & BCC (sclerodermiform) & 87 & $\mathrm{~F}$ & Nose & $2+$ & $3+$ & $3+$ & $3+$ \\
\hline 29 & BCC (sclerodermiform) & 81 & $\mathrm{~F}$ & Nose & $2+$ & $3+$ & $3+$ & $3+$ \\
\hline 30 & BCC (sclerodermiform) & 78 & $\mathrm{M}$ & Ear & $1+$ & $3+$ & $3+$ & $3+$ \\
\hline 31 & BCC (baso-squamous) & 85 & $\mathrm{M}$ & Forehead & $1+$ & $3+$ & $3+$ & $3+$ \\
\hline 32 & BCC (baso-squamous) & 54 & $\mathrm{~F}$ & Periocular & $2+$ & $3+$ & $3+$ & $3+$ \\
\hline 33 & BCC (baso-squamous) & 80 & $\mathrm{M}$ & Neck & $3+$ & $3+$ & $3+$ & $3+$ \\
\hline 34 & BCC (baso-squamous) & 78 & $\mathrm{~F}$ & Leg & $2+$ & $3+$ & $3+$ & $3+$ \\
\hline
\end{tabular}

BCC, basal cell carcinoma; SCC, squamous cell carcinoma; 0, negative staining; $1+, 1-20 \%$ of positive cells; $2+, 21-60 \%$ of positive cells; $3+$, more than $60 \%$ of positive cells.

incubation with the secondary antibody (UltraVision kit; LabVision). Bound antibodies were visualized using 3'-3-diaminobenzidine as chromogen. Nuclei were slightly counterstained with Mayer's hematoxylin. Negative controls were performed by substituting the primary antibody with a non-immune serum. Control sections were treated in parallel with the samples, in the same run. Immunostained sections were independently assessed by three observers (DM, JP, MS). The results were expressed according to semiquantitative criteria as follows: $0=$ negative staining; $1+=1-20 \%$ of positive cells; $2+=21-60 \%$ of positive cells and $3+=$ more than $60 \%$ of positive cells. The staining intensity was scored on a scale as faint, moderate or strong. Owing to the small number of samples, no statistical analysis of the results was conducted.

Two cases of Bowen's disease and all squamous cell carcinomas on genital site were evaluated by in situ hybridization for human papilloma virus (HPV) with an automatic procedure (Benchmark Ventana, Tucson, AZ, USA). Two DNA probes were used: (i) Ventana inform HPV high risk (cocktail of HPV genomic probes for 6 and 11 genotypes) and (ii)
Ventana inform HPV low risk (cocktail of HPV genomic probes for $16,18,31,33,35,39,45,51$, $52,56,58,59,68$ and 70 genotypes). Signals were detected with a Ventana iView Blue Detection kit. Staining was considered positive when cell nuclei were unequivocally stained blue.

The intensity of Notch 1 staining was compared between different types of cutaneous lesions. For statistical analysis, a $\chi^{2}$ test was used. A $P$-value less than 0.05 was considered statistically significant.

\section{Results}

Notch 1 immunohistochemical expression was mostly confined to the cell cytoplasm, with occasional peripheral membrane staining and nuclear pattern. Evaluation of Jagged 1, Jagged 2 and Deltalike 1 immunoreactions displayed predominantly cytoplasmic and plasma membrane staining in either normal or neoplastic cells, and no consistent nuclear pattern was observed. In normal skin, Notch 1 was strongly and diffusely expressed in suprabasal epidermal keratinocytes, with only faint and focal 
staining in the granular layer and stratum corneum. A high expression level of Notch 1 was consistently observed in adnexal structures, including hair follicle epithelium, sweat and sebaceous glands. Jagged 1, Jagged 2 and Delta-like 1 were diffusely expressed in suprabasal keratinocytes, up to the granular cell layer, as well as in adnexal structures, although more weakly.

Notch 1 expression was heterogeneous within examined skin samples, while Jagged 1, Jagged 2 and Delta-like 1 immunostaining was diffusely positive in all cases. In photoaged skin, characterized by massive accumulation of abnormal elastic fibers (so-called solar elastosis), dilated dermal capillary vessels and sparse lymphocyte infiltrates, the overlying epidermis showed Notch 1 positivity in both basal and suprabasal layers (Figure 1a), even though there were some focal areas of normal-appearing epidermis characterized by Notch 1 downregulation.
In solar keratoses, atypical keratinocytes with hyperchromic nuclei showed a partial to complete loss of Notch 1 expression (Figure 1b), while the basal cell layer was still Notch 1 immunoreactive. Atypical keratinocytes, spreading into the infundibular and isthmic segments of follicles or along eccrine ducts, were also Notch 1 negative. No differences in Notch 1 expression were observed between atrophic and hypertrophic types of solar keratoses. Early UV-induced squamous cell carcinomas on sun-exposed skin, in which detachment of individual, irregular aggregates of atypical squamous cells in the superficial dermis was detected, downregulated Notch 1 protein (Figure 1c) similarly to nests, sheets and strands of squamous epithelial cells extending in the deep dermis in clearly invasive UV-induced squamous cell carcinomas (Figure 1d). No differences in Notch 1 staining were noted according to the grade of differentiation.
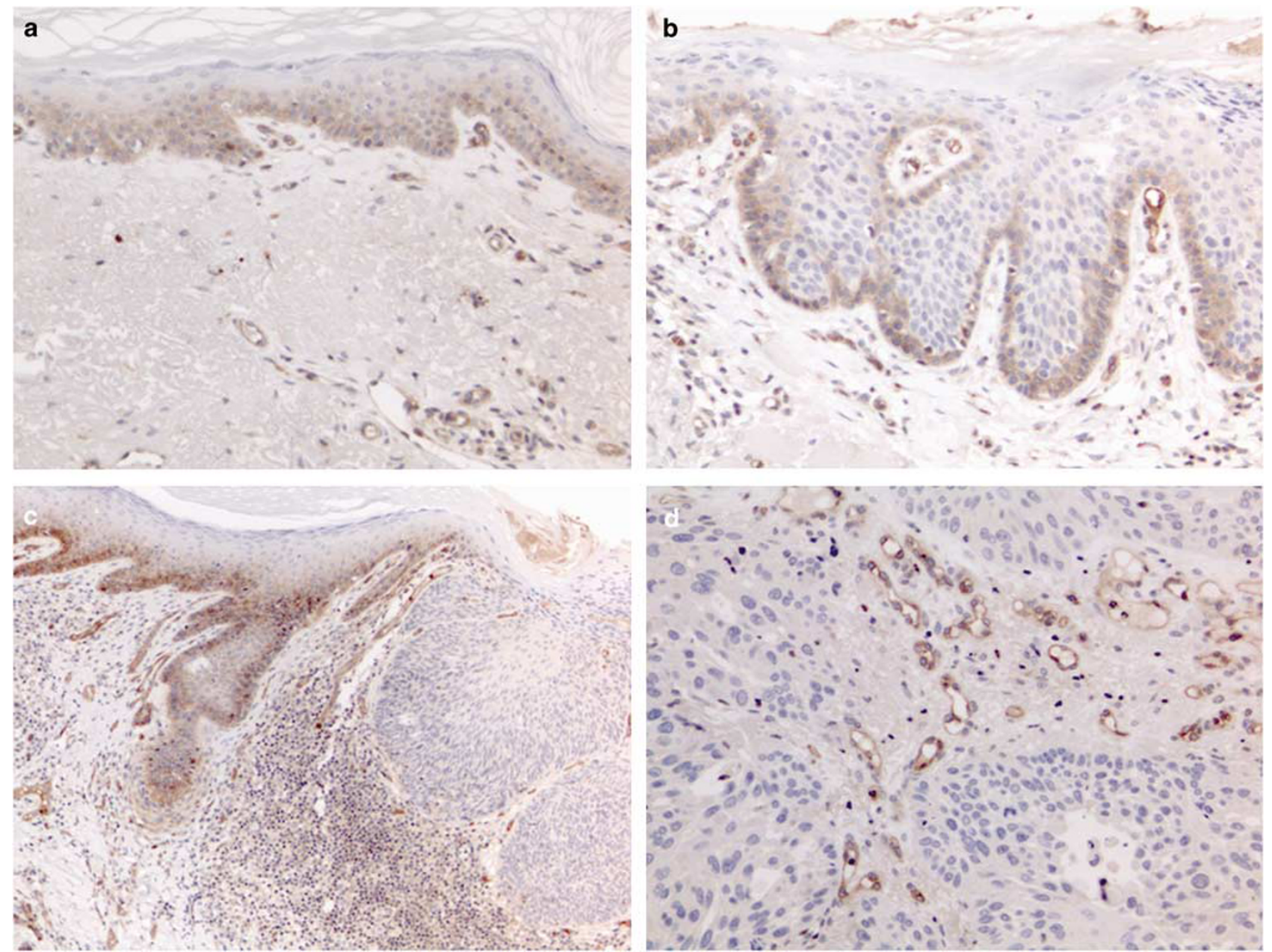

Figure 1 Photodamaged skin shows diffuse Notch 1 expression in both basal and suprabasal epidermal layers (a). In solar keratosis, buds of atypical squamous cells with hyperchromic nuclei show loss of Notch 1 expression (b), while the basal cell layer is Notch 1 immunoreactive. Early UV-induced squamous cell carcinomas on sun-exposed site, characterized by detachment of individual, irregular aggregates of atypical squamous cells in the superficial dermis, downregulates Notch 1 protein expression (c) similarly to sheets and strands of squamous atypical epithelial cells, extending in the deep dermis, in clearly invasive UV-induced squamous cell carcinomas (d). 
The epidermis in Bowen's disease showed loss of polarity and a crowding of atypical keratinocytes with hyperchromatism, pale-staining to vacuolated cells, occasional multinucleated cells, confined to the dermo-epidermal junction (in situ squamous cell carcinoma) (Figure 2a). All cases of Bowen's disease were diffusely Notch 1 positive, irrespective from the location (extremities vs face vs trunk). In two lesions, we observed vacuolated atypical cells, highly suggestive of koilocytotic viral cytopathic change, in pagetoid spread. These lesions, however, resulted HPV negative by in situ hybridization. HPVpositive squamous cell carcinomas arising on sunprotected penile skin were all diffusely Notch 1 positive, in both in situ (Figure 2b) and invasive components (Figure 2c). In one of these cases, a strong and diffuse Notch 1 membrane pattern was observed (Figure 2d).

In basal cell carcinomas, differential Notch 1 expression was found according to subtypes. All nodular (Figure 3a) and superficial type basal cell carcinomas (Figure 3b) displayed a strong diffuse Notch 1 positivity within lobules of basaloid neoplastic cells. Conversely, strands, cords and columns of basaloid cells with scant cytoplasm surrounded by marked fibrosis, typical of sclerodermiform/infiltrating basal cell carcinomas, showed low-to-moderate Notch 1 expression (Figure 3c). Finally, in most baso-squamous basal cell carcinomas, neoplastic cells characterized by a more abundant cytoplasm and evidence of keratinization displayed a heterogeneous staining with areas of Notch 1 negativity (Figure 3d).

Statistical analysis of Notch 1 staining showed statistically significant differences in different categories of skin lesions, including solar keratoses vs Bowen's disease, $P<0.007$; sun-exposed squamous cell carcinoma vs sun-protected squamous cell carcinoma, $P<0.001$; basal cell carcinoma (nodular + superficial type) vs basal cell carcinoma (sclerodermiform + baso-squamous type), $P<0.043$. We found a heterogeneous p53 expression in the skin samples examined (Table 2). In particular, there was an increase of p53 labeling from non-sunexposed to sun-exposed skin (Figure 4a), solar keratoses (Figure 4b) and UV-induced squamous
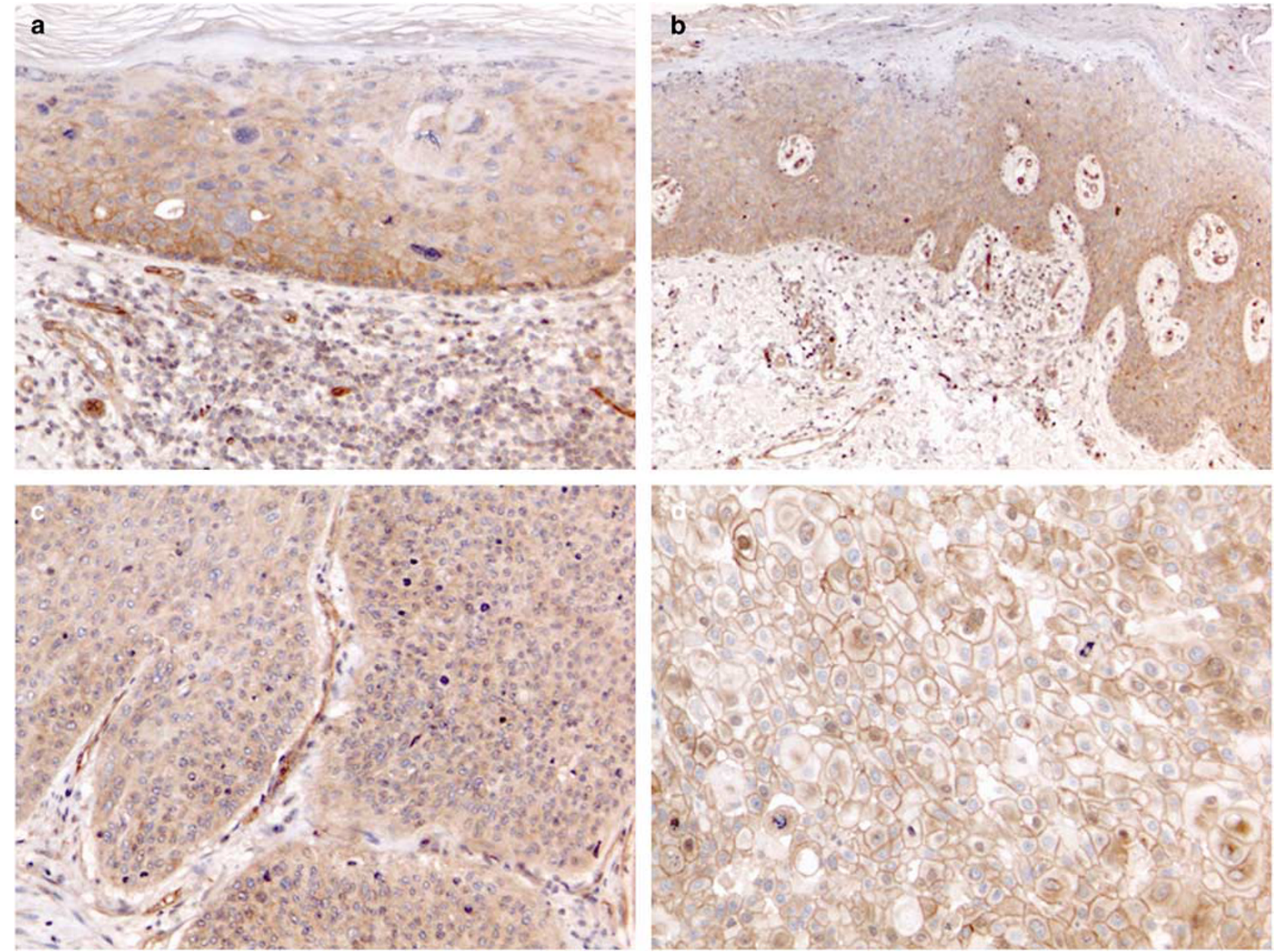

Figure 2 Notch 1 is diffusely positive in the epidermis of Bowen's disease (a), irrespective from location (extremities vs face vs trunk). HPV-positive squamous cell carcinomas arising in sun-protected penile skin were all Notch 1 diffusely positive, in both in situ (b) and invasive component (c). Strong and diffuse Notch 1 membrane pattern in an invasive squamous cell carcinomas in sun-protected site (d). 

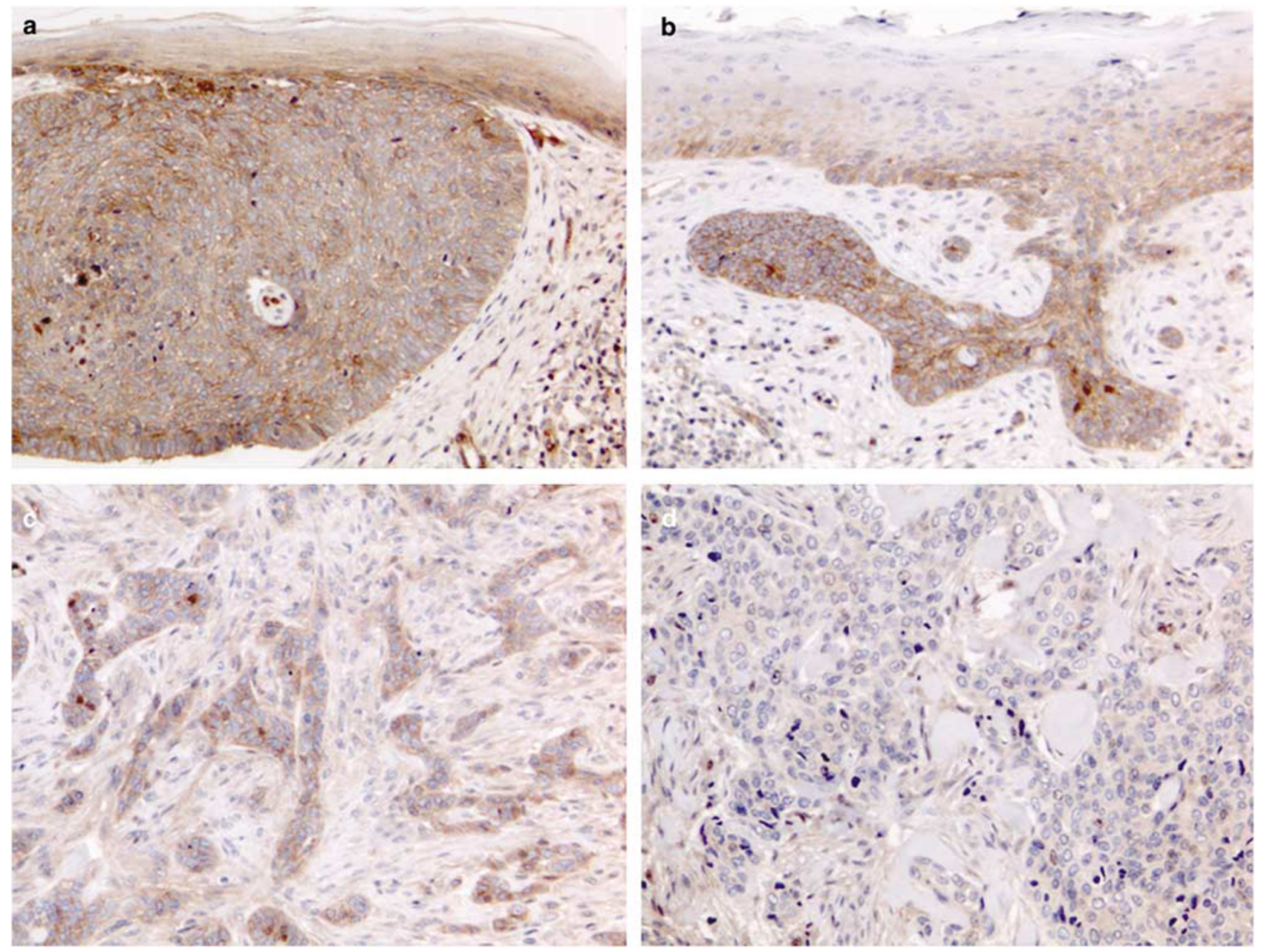

Figure 3 Nodular (a) and superficial type basal cell carcinomas (b) display strong and diffuse Notch 1 positivity within lobules of basaloid neoplastic cells. Strands and cords of basaloid cells with scant cytoplasm surrounded by marked fibrosis, typical of sclerodermiform/infiltrating basal cell carcinoma, show low Notch 1 expression (c). Absence of Notch 1 immunostaining in basosquamous basal cell carcinoma (d).

Table 2 Distribution of p53 expression in solar keratoses, Bowen's disease, squamous cell carcinoma and basal cell carcinoma $(n=15)$

\begin{tabular}{rlclll}
\hline No. & Diagnosis & Age (years) & Sex & Site & p53 \\
\hline 1 & Solar keratosis & 61 & $\mathrm{~F}$ & Nose & $1+$ \\
2 & Solar keratosis & 79 & $\mathrm{M}$ & Forearm & $3+$ \\
7 & Bowen's disease & 53 & $\mathrm{M}$ & Leg & $1+$ \\
8 & Bowen's disease & 89 & $\mathrm{M}$ & Thigh & $1+$ \\
10 & SCC (sun-exposed site) & 92 & $\mathrm{M}$ & Cheek & $3+$ \\
12 & SCC (sun-exposed site) & 91 & $\mathrm{M}$ & Scalp & $3+$ \\
13 & SCC (sun-exposed site) & 82 & $\mathrm{~F}$ & Nose & $3+$ \\
17 & SCC (sun-protected site) & 93 & $\mathrm{M}$ & Penis & $2+$ \\
18 & SCC (sun-protected site) & 72 & $\mathrm{M}$ & Penis & $1+$ \\
19 & SCC (sun-protected site) & 81 & $\mathrm{M}$ & Penis & $2+$ \\
20 & SCC (sun-protected site) & 43 & $\mathrm{M}$ & Penis & $1+$ \\
22 & BCC (nodular type) & 73 & $\mathrm{~F}$ & Face & $2+$ \\
27 & BCC (superficial type) & 66 & $\mathrm{M}$ & Back & $2+$ \\
28 & BCC (sclerodermiform) & 87 & $\mathrm{~F}$ & Nose & $3+$ \\
30 & BCC (sclerodermiform) & 78 & $\mathrm{M}$ & Ear & $3+$ \\
& & & & & \\
\hline
\end{tabular}

BCC, basal cell carcinoma; SCC, squamous cell carcinoma; 0, negative staining; $1+, 1-20 \%$ of positive cells; $2+, 21-60 \%$ of positive cells; $3+$, more than $60 \%$ of positive cells. cell carcinomas (Figure 4c). Conversely, most cases of Bowen's disease displayed low p53 expression (Figure 5a); well-differentiated invasive squamous cell carcinomas (Figure 5b) and less differentiated invasive squamous cell carcinomas on sun-protected sites (Figure 5c) expressed moderate p53 protein levels when compared with the high p53 expression in UV-related invasive squamous cell carcinomas (Figure 5d).

\section{Discussion}

In the present study, we showed a differential expression of Notch 1 protein in non-melanoma skin cancer. Notch 1 is downregulated in solar keratoses and invasive squamous cell carcinomas arising on sun-exposed sites, suggesting a tumor suppressor effect of Notch signaling in UV-related squamous cell photocarcinogenesis. In contrast, on sun-protected HPV-related squamous cell 

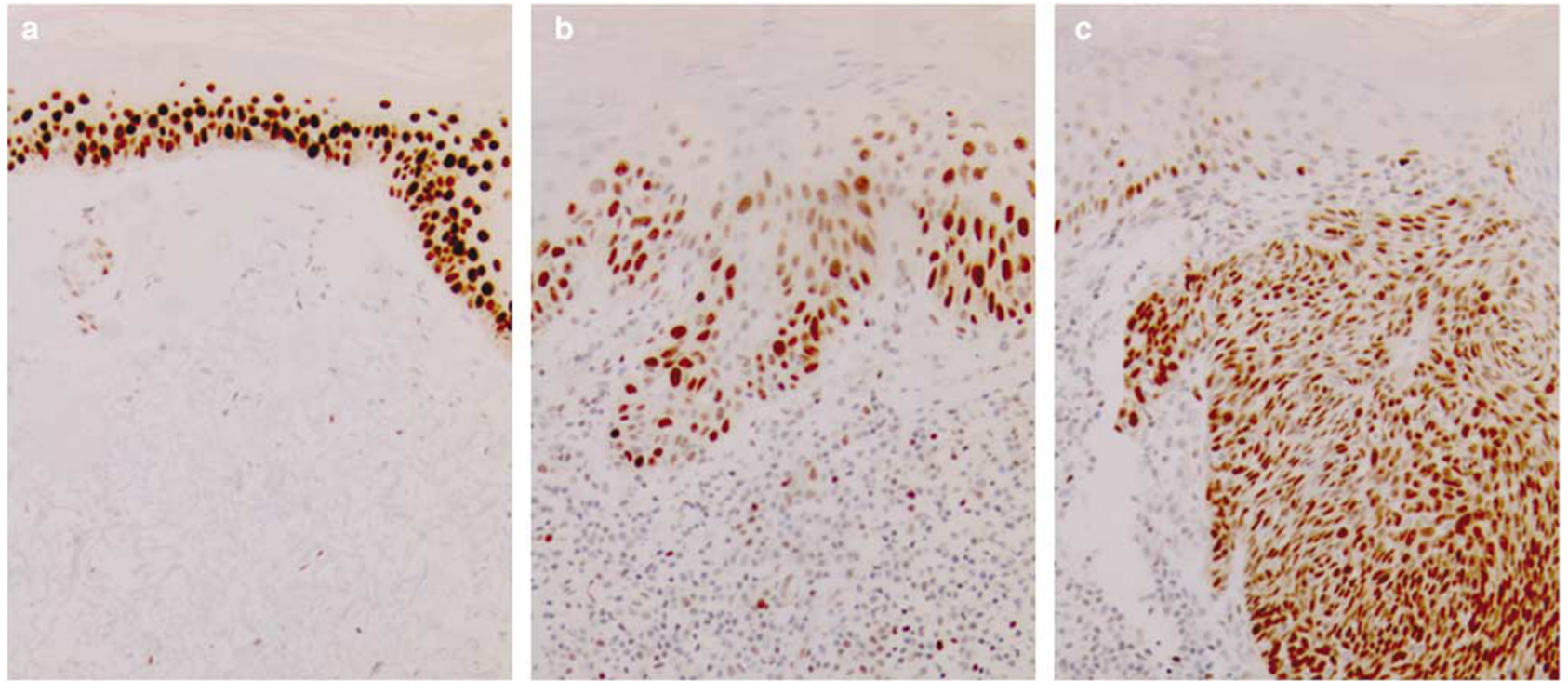

Figure 4 Consistent p53 labeling in sun-exposed skin (a), solar keratoses (b) and UV-induced squamous cell carcinomas (c).
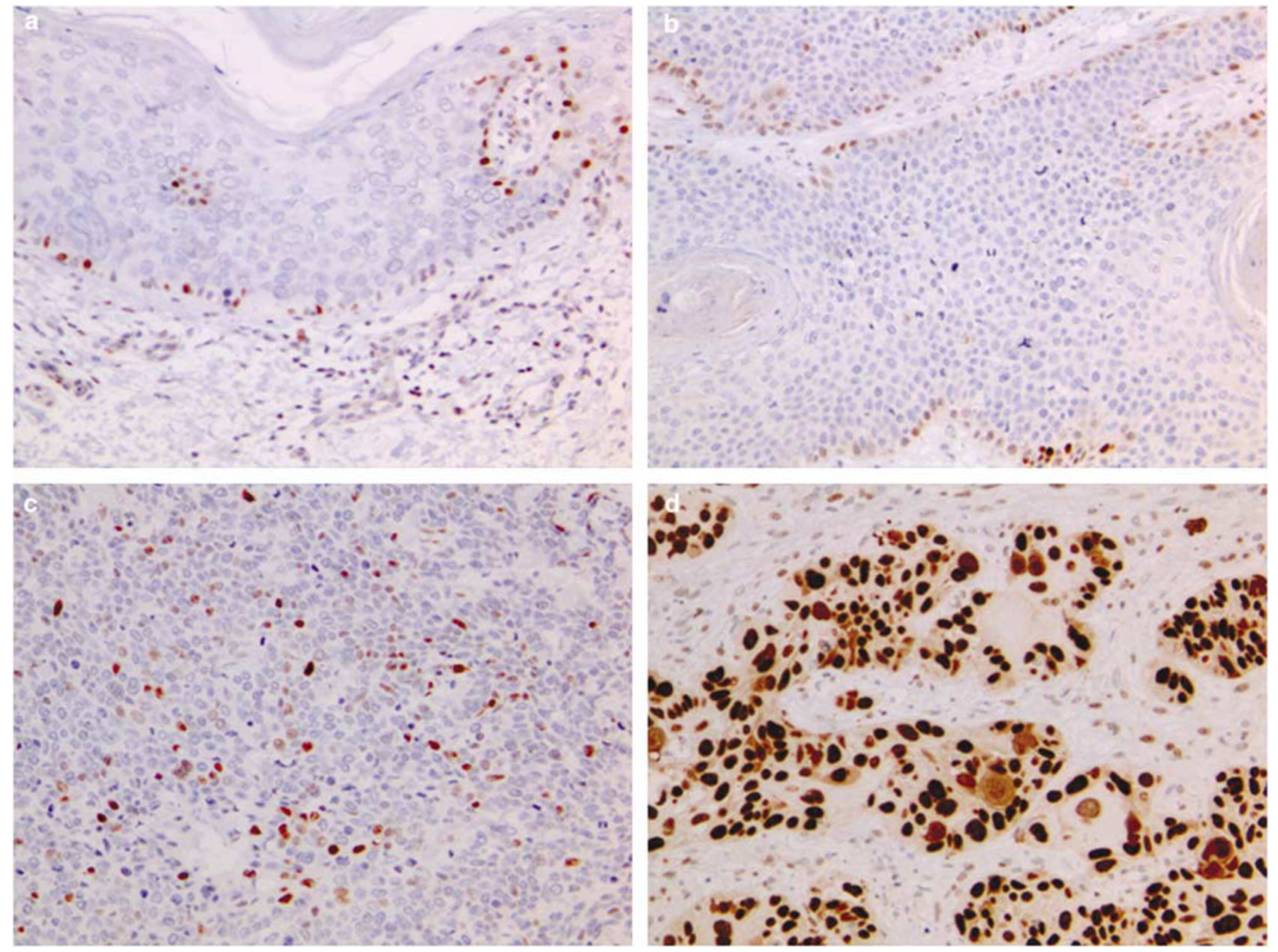

Figure 5 Bowen's disease displays low p53 expression (a). Well-differentiated invasive squamous cell carcinomas on sun-protected site (b) and less differentiated invasive squamous cell carcinomas on sun-protected site (c) express moderate p53 protein levels when compared with the high p53 expression in UV-related invasive squamous cell carcinomas on sun-exposed location (d). 
carcinomas (arising on genital skin), Notch 1 is upregulated. Furthermore, Notch 1 is diffusely expressed in the common nodular and superficialtype basal cell carcinomas, while its expression is decreased in subtypes correlated with higher risk of recurrences and metastases (sclerodermiform/infiltrating and baso-squamous types). Thus, our data support the notion that Notch signaling has varied roles in non-melanoma skin cancer that reflect its versatile functions in development and cancer tissue homeostasis. Although the precise mechanism for this dual action of Notch remains to be explored, our findings suggest that UV radiations may play a major role in determining its oncosuppressive function.

Previous studies strongly support a tumor suppression effect of Notch 1 signaling in non-melanoma skin cancer. In mouse experimental models, it has been shown that Notch 1 ablation in epidermis causes hyperplasia and skin tumors and facilitates chemical-induced skin carcinomas. ${ }^{14}$ Interestingly, given the simultaneous development of different types of skin lesions (papillomas, dysplastic lesions, basal cell carcinoma-like tumors and squamous cell carcinomas), it was suggested that tumor type may be dependent on quality and quantity of additional acquired mutations. ${ }^{14}$ Subsequently, Proweller et $a l^{15}$ showed that the transgenic DNMAML1 mice, expressing a dominant-negative MAML1 (DNMAML1) protein that inhibits CSL-dependent Notch signaling in the epidermis, exhibited multiple skin defects, including squamous cell carcinomalike tumors and solar keratoses, but did not develop basal cell carcinomas.

Our observations suggest that cutaneous squamous cell carcinomas on sun-exposed sites downregulate Notch 1, possibly as a consequence of p53 gene mutations, induced by UV radiations. In line with this hypothesis, previous in vitro studies have shown that Notch 4 mRNA expression is significantly downregulated in keratinocytes after UVB treatment. These data suggest that Notch 1 may influence the epidermal microenvironment after sunlight exposure. ${ }^{16}$ UVB exposure induces DNA thymidine dimer formation, which can target p53, with impaired apoptosis of damaged keratinocytes in cells with two p53 mutations. ${ }^{17,18}$ Overall, solar keratoses carry p53 mutations in about $60 \%$ of cases, $89 \%$ of which are typical UV type mutations. ${ }^{19}$ Solar keratoses also display gene mutations of Kras, although ras oncogenes do not appear to be as important as $p 53$ in skin cancer development. ${ }^{20}$ Therefore, a combination of these genetic abnormalities might be crucial to carcinogenesis, at least in a subset of squamous cell carcinomas.

The relationship between UV radiations, Notch signaling and p53 in the context of skin cancer is a complex and yet not fully clarified issue. It has been recently shown that Notch 1 gene is a downstream positive target of p53, which acts in concert with other key regulatory proteins. ${ }^{12}$ On the other hand, expression of Notch 1 IC downregulates p53responsive genes, $p 21, m d m 2$ and bax, in HCT116 p53(-/-) cells. $^{21}$ In addition, activated Notch 1 interacts with $p 53$ to inhibit its phosphorylation and transactivation and downregulates p53-dependent apoptosis induced by UV irradiation. ${ }^{21}$

In tissue specimens, we found an inverse correlation between p53 and Notch 1 expression, with an increase in p53 labeling from non-sun-exposed skin to sun-exposed skin, solar keratoses and UV-induced squamous cell carcinomas and a higher p53 expression in sun-exposed squamous cell carcinomas in comparison with squamous cell carcinomas arising on sun-protected skin. Previous studies indicate a wide variation and heterogeneity of p53 expression in photodamaged skin and nonmelanoma skin cancer. Discrepancies may be due to the severity of tissue damage, cumulative (chronic) and acute sun exposure, and host/genetic factors such as DNA repair capacity. ${ }^{22}$

Unexpectedly, Bowen's disease and squamous cell carcinomas arising on sun-protected genital skin were found to be Notch 1 positive. Squamous cell carcinomas arising on genital sites are commonly associated with HPV infection, thus we hypothesize that Notch expression in this setting may be triggered by viral infection. In HPV 16- and 18-induced genital squamous cell carcinomas, viral E6 protein binds to p53 and E7 binds to Rb protein. The inhibition of these tumor suppessor genes induces oncogenesis. In the literature, however, there are apparently contradictory results concerning HPV-related oncogenes and Notch signaling in tumors. In cervical cancer, expression of HPV oncoproteins E6/E7 alone is insufficient to transform human epithelial cells, and it has been recently demonstrated that coexpression of Notch IC complements E6/E7 activity in fully transformed cells. ${ }^{23,24}$ In contrast, Talora et $a l^{25}$ reported that increased signaling by Notch 1 causes downmodulation of HPV-driven transcription of E6/E7 viral genes. In a later study, Talora et $a{ }^{26}$ showed that in HPV-positive cervical cancer cells, activated Notch 1 causes growth suppression. Since these latter data were obtained using an adenovirus-mediated Notch 1 overexpression strategy, it remains uncertain whether spontaneously expressed Notch 1 decreases viral oncoprotein load in HPV-positive cervical carcinoma cells. Recently, it has been shown that increased Notch 1 signaling induced a downmodulation of HPV transcription by upregulation of $C$-Jun and downregulation of $c-F_{O S}{ }^{27}$ Thus, it seems that concentration/dose, tissue context and copresence of other oncogenes may affect the net outcome of Notch signaling.

Another interesting outcome of our study is the differential Notch 1 expression according to basal cell carcinoma histotype. In previous studies, Nicolas et $a 1^{14}$ demonstrated that Notch 1 deficiency in mouse skin results in increased and sustained expression of Gli2, causing the development of basal 
cell carcinoma-like tumors. Thelu et $\left.a\right|^{28}$ reported that transcript levels of Notch 1-3 and their ligands detected by in situ hybridization were severely lowered in basal cell carcinoma tissue samples, with no expression detected in deep nodular and infiltrative basal cell carcinomas.

What it is not yet clear is whether functional inactivation or loss of Notch 1 is necessary for basal cell carcinoma progression in humans. Preliminary observations suggest that basal cell carcinomas lack activated Notch, consistent with the putative tumorsuppressive role of the receptor in the epidermis. ${ }^{29}$ Suppression of Notch 1 was hypothesized to be related to growth promotion with an unknown mechanism, although data obtained in invertebrate models suggest that it may be related to the activation of the Wnt/Wingless pathway. ${ }^{29}$ Overexpression of Wnt ligands in basal cell carcinomas may be triggered by PATCH mutations, which are known to play a key role in basal cell carcinoma pathogenesis. ${ }^{30,31}$

In conclusion, our data provide new insights into the molecular mechanisms involved in the development and regulation of non-melanoma skin cancer and point to a crucial role of UV radiations in determining Notch 1 downmodulation in human squamous cell carcinoma, since the early stages of UV-induced skin cancerogenesis.

\section{Acknowledgement}

This study was financially supported by Fondazione Ente Cassa di Risparmio di Firenze.

\section{References}

1 Weinstock MA. Epidemiology of nonmelanoma skin cancer: clinical issues, definitions, and classification. J Invest Dermatol 1994;102:4S-5S.

2 Alam M, Ratner D. Cutaneous squamous-cell carcinoma. N Engl J Med 2001;344:975-983.

3 Artavanis-Tsakonas S, Rand MD, Lake RJ. Notch signaling: cell fate control and signal integration in development. Science 1999;284:770-776.

4 Gray GE, Mann RS, Mitsiadis E, et al. Human ligands of the Notch receptor. Am J Pathol 1999;154:785-794.

5 Shutter JR, Scully S, Fan W, et al. Dll4, a novel Notch ligand expressed in arterial endothelium. Genes Dev 2000;14:1313-1318.

6 Schroeter EH, Kisslinger JA, Kopan R. Notch-1 signalling requires ligand-induced proteolytic release of intracellular domain. Nature 1998;393:382-386.

7 Levitan D, Lee J, Song L, et al. PS1 N- and C-terminal fragments form a complex that functions in APP processing and Notch signaling. Proc Natl Acad Sci USA 2001;98:12186-12190.

8 Miele L, Golde T, Osborne B. Notch signaling in cancer. Curr Mol Med 2006;6:905-918.

9 Massi D, Tarantini F, Franchi A, et al. Evidence for differential expression of Notch receptors and their ligands in melanocytic nevi and cutaneous malignant melanoma. Mod Pathol 2006;19:246-254.

10 Rangarajan A, Talora C, Okuyama R, et al. Notch signaling is a direct determinant of keratinocyte growth arrest and entry into differentiation. EMBO J 2001;20:3427-3436.

11 Nickoloff BJ, Qin JZ, Chaturvedi V, et al. Jagged-1 mediated activation of notch signaling induces complete maturation of human keratinocytes through NF-kappaB and PPARgamma. Cell Death Differ 2002;9: 842-855.

12 Lefort K, Mandinova A, Ostano P, et al. Notch1 is a p53 target gene involved in human keratinocyte tumor suppression through negative regulation of ROCK1/2 and MRCKalpha kinases. Genes Dev 2007;21:562-577.

13 Devgan V, Mammucari C, Millar SE, et al. p21WAF1/ Cip1 is a negative transcriptional regulator of Wnt4 expression downstream of Notch1 activation. Genes Dev 2005;19:1485-1495.

14 Nicolas M, Wolfer A, Raj K, et al. Notch1 functions as a tumor suppressor in mouse skin. Nat Genet 2003;33: $416-421$.

15 Proweller A, Tu L, Lepore JJ, et al. Impaired notch signaling promotes de novo squamous cell carcinoma formation. Cancer Res 2006;66:7438-7444.

16 Murakami T, Fujimoto M, Ohtsuki M, et al. Expression profiling of cancer-related genes in human keratinocytes following non-lethal ultraviolet B irradiation. J Dermatol Sci 2001;27:121-129.

17 Basset-Seguin N, Moles JP, Mils V, et al. TP53 tumor suppressor gene and skin carcinogenesis. J Invest Dermatol 1994;103(5 Suppl):102S-106S.

18 Ziegler A, Jonason AS, Leffell DJ, et al. Sunburn and p53 in the onset of skin cancer. Nature 1994;372: 773-776.

19 Benjamin CL, Ananthaswamy HN. p53 and the pathogenesis of skin cancer. Toxicol Appl Pharmacol 2007;224:241-248.

20 Boukamp P. Non-melanoma skin cancer: what drives tumor development and progression? Carcinogenesis 2005;26:1657-1667.

21 Kim SB, Chae GW, Lee J, et al. Activated Notch1 interacts with p53 to inhibit its phosphorylation and transactivation. Cell Death Differ 2007;14:982-991.

22 Einspahr J, Alberts DS, Aickin M, et al. Expression of p53 protein in actinic keratosis, adjacent, normalappearing, and non-sun-exposed human skin. Cancer Epidemiol Biomarkers Prev 1997;6:583-587.

23 Lathion S, Schaper J, Beard P, et al. Notch1 can contribute to viral-induced transformation of primary human keratinocytes. Cancer Res 2003;63: 8687-8694.

24 Weijzen S, Zlobin A, Braid M, et al. HPV16 E6 and E7 oncoproteins regulate Notch-1 expression and cooperate to induce transformation. J Cell Physiol 2003;194:356-362.

25 Talora C, Sgroi DC, Crum CP, et al. Specific downmodulation of Notch1 signaling in cervical cancer cells is required for sustained HPV-E6/E7 expression and late steps of malignant transformation. Genes Dev 2002;16:2252-2263.

26 Talora C, Cialfi S, Segatto O, et al. Constitutively active Notch1 induces growth arrest of HPV-positive cervical cancer cells via separate signaling pathways. Exp Cell Res 2005;305:343-354.

27 Wang L, Qin H, Chen B, et al. Overexpressed active Notch1 induces cell growth arrest of HeLa 
cervical carcinoma cells. Int J Gynecol Cancer 2007;17: 1283-1292.

28 Thelu J, Rossio P, Favier B. Notch signalling is linked to epidermal cell differentiation level in basal cell carcinoma, psoriasis and wound healing. BMC Dermatol 2002;2:7.

29 Nickoloff BJ, Osborne BA, Miele L. Notch signaling as a therapeutic target in cancer: a new approach to the development of cell fate modifying agents. Oncogene 2003;22:6598-6608.

30 Gailani MR, Bale AE. Acquired and inherited basal cell carcinomas and the patched gene. Adv Dermatol 1999;14:261-283.

31 Holikova Z, Massi D, Lotti T, et al. Insight into the pathogenesis of sporadic basal cell carcinoma. Int J Dermatol 2004;43:865-869. 